Two-Dimensional Organoferroelasticity in a Single Crystal of 4-Iodoaniline

Subham Ranjan ${ }^{\mathrm{a}}$ and Satoshi Takamizawa ${ }^{\text {a* }}$

a Department of Materials System Science, Graduate School of Nanobioscience,

Yokohama City University, 22-2 Seto, Kanazawa-ku, Yokohama, Kanagawa 236-0027 (Japan)

E-mail:staka@yokohama-cu.ac.jp

Table of contents:

Page

Thermal analysis

S2

Crystallographic studies

S2-S6

\title{
Other supporting material
}

Ferroelastic behavior under the microscope by shearing with tweezers at room temperature

Movie S1. Shearing with tweezers on (100) plane (MOV)

Movie S1. Shearing with tweezers on $(00 \overline{1})$ plane (MOV)

Ferroelastic behavior under the microscope during force measurement at room temperature

Movie S3. Stress-displacement test on (100) plane (MOV)

Movie S4. Stress-displacement test on $(00 \overline{1})$ plane (MOV) 
(a)

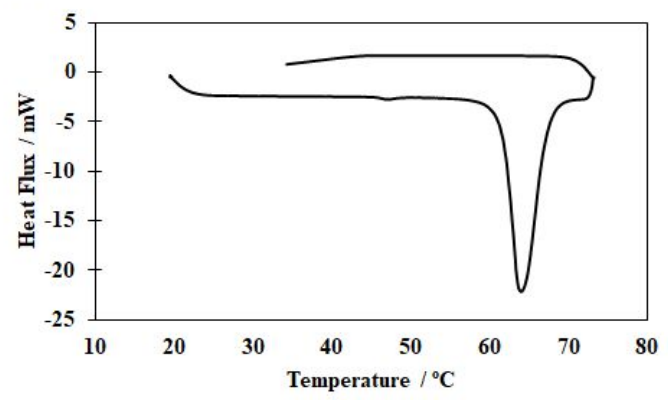

(b)

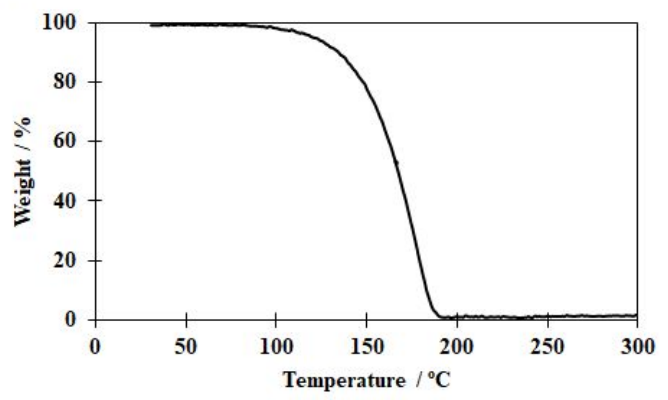

Figure S1. (a) DSC curve and (b) Thermogravimetric profile of 4-Iodoaniline.

Table S1. Crystallographic data of 4-Iodoaniline

\begin{tabular}{|c|c|c|c|}
\hline Domain & $\alpha_{M}$ & $\alpha_{\mathrm{D} 1}$ & $\alpha_{\mathrm{D} 2}$ \\
\hline $\mathrm{T} / \mathrm{K}$ & $203(2)$ & $203(2)$ & $203(2)$ \\
\hline Empirical formula & $\begin{array}{l}\mathrm{C}_{6} \mathrm{H}_{6} \mathrm{IN} \text { (Mother } \\
\text { Domain) }\end{array}$ & $\begin{array}{l}\mathrm{C}_{6} \mathrm{H}_{6} \mathrm{IN} \text { (Daughter } \\
\text { Domain 1) }\end{array}$ & $\begin{array}{l}\mathrm{C}_{6} \mathrm{H}_{6} \mathrm{IN} \text { (Daughter } \\
\text { Domain 2) }\end{array}$ \\
\hline Crystal system & Monoclinic & Monoclinic & Monoclinic \\
\hline Space group & $P 2_{1}$ & $P 2_{1}$ & $P 2_{1}$ \\
\hline$a / \AA$ & $8.4478(4)$ & 8.464 & $8.4501(7)$ \\
\hline$b / \AA$ & $4.9720(2)$ & 4.983 & $4.9724(4)$ \\
\hline$c / \AA$ & $8.6846(4)$ & 8.683 & $8.6791(7)$ \\
\hline$\alpha /{ }^{\circ}$ & 90 & 90 & 90 \\
\hline$\beta /{ }^{\circ}$ & $109.8600(10)$ & 109.86 & $109.872(2)$ \\
\hline$\gamma /{ }^{\circ}$ & 90 & 90 & 90 \\
\hline $\mathrm{V} / \AA ̊ 3$ & $343.08(3)$ & 344.4 & $342.96(5)$ \\
\hline Z & 2 & 2 & 2 \\
\hline$\rho_{\text {calcd }}\left[\mathrm{g} \mathrm{cm}^{-3}\right]$ & 2.120 & 2.112 & 2.121 \\
\hline$F(000)$ & 204 & 204 & 204 \\
\hline
\end{tabular}




\begin{tabular}{|l|l|l|l|}
\hline$\mu\left[\mathrm{mm}^{-1}\right]$ & 4.560 & 4.542 & 4.562 \\
\hline index ranges & $\begin{array}{l}-10 \leq \mathrm{h} \leq 9,-5 \leq \mathrm{k} \\
\leq 5,-9 \leq 1 \leq 10\end{array}$ & $\begin{array}{l}-10 \leq \mathrm{h} \leq 9,-5 \leq \mathrm{k} \leq \\
5,-9 \leq 1 \leq 10\end{array}$ & $\begin{array}{l}-10 \leq \mathrm{h} \leq 9,-5 \leq \mathrm{k} \leq 5,- \\
9 \leq 1 \leq 10\end{array}$ \\
\hline $\begin{array}{l}\text { Reflections } \\
\text { collected }\end{array}$ & 9972 & 5830 & 2835 \\
\hline Goodness of fit & 1.092 & 1.150 & 1.172 \\
\hline$R_{1}(I>2 \sigma$ (all data) $)$ & 0.0305 & 0.0276 & 0.0296 \\
\hline $\begin{array}{l}\text { wR } R_{2}(I>2 \sigma \text { (all } \\
\text { data) })\end{array}$ & 0.0941 & 0.0875 & 0.0739 \\
\hline CCDC No. & 2124588 & 2124589 & 2124590 \\
\hline
\end{tabular}

(a)

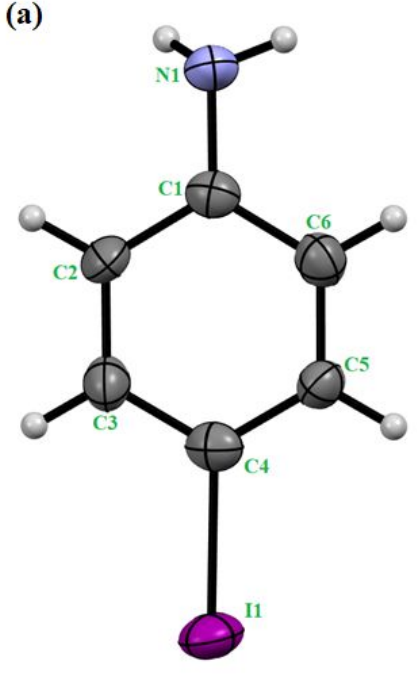

(b)

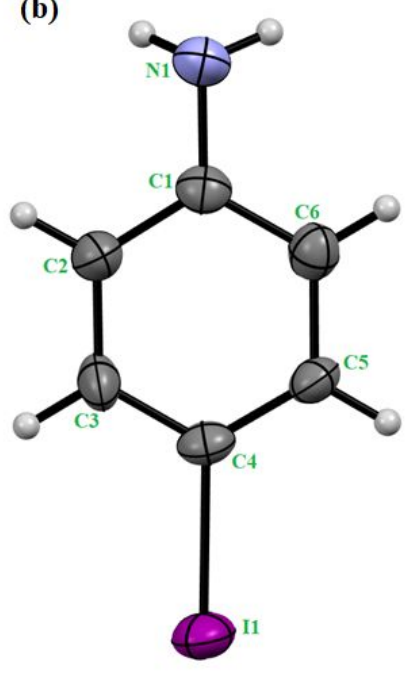

(c)

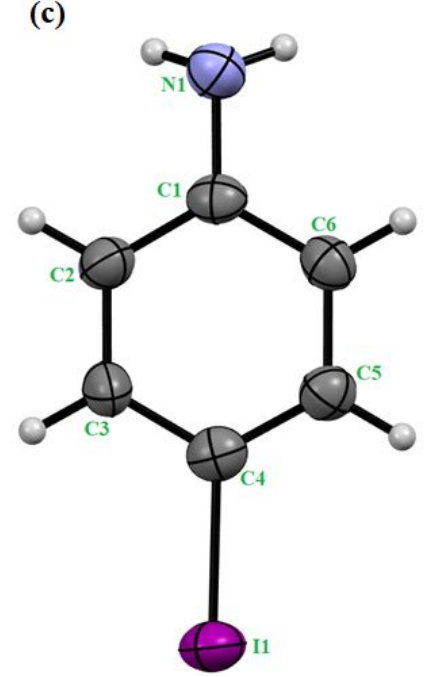

Figure S2. ORTEP representation of (a) Mother domain $\left(\alpha_{M}\right)$, (b) Daughter domain $\left(\alpha_{D 1}\right)$, and (d) Daughter domain $\left(\alpha_{\mathrm{D} 2}\right)$, where the displacement ellipsoids were drawn at 50\% probability level. 


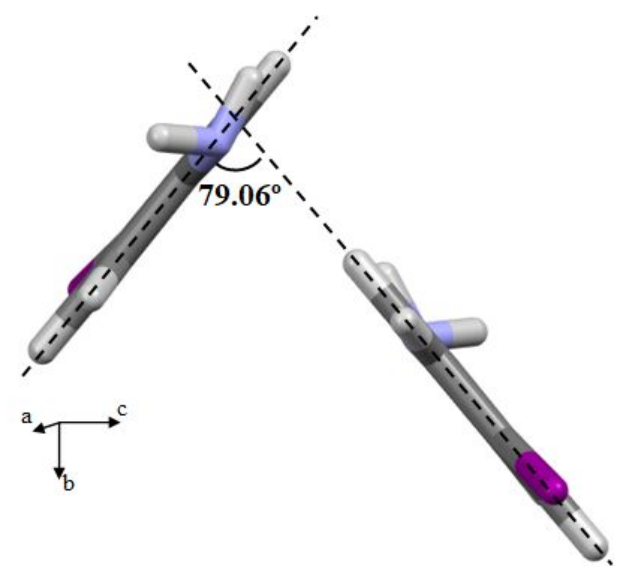

Figure S3. Dihedral angles between the planes passing through benzene ring $(C(1)-C(2)-C(3)-$ $C(4)-C(5)-C(6))$ of each molecule.
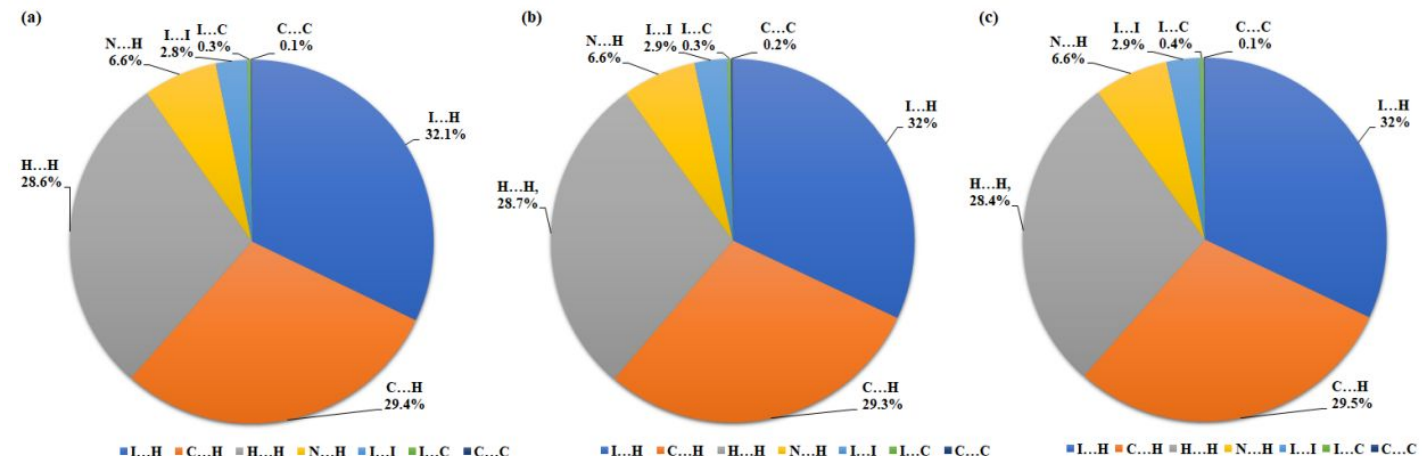

Figure S4. Relative contributions of intermolecular interactions of 4-Iodoaniline. (a) Mother domain $\left(\alpha_{M}\right)$, (b) Daughter domain $\left(\alpha_{D 1}\right)$, and (d) Daughter domain $\left(\alpha_{D 2}\right)$. 


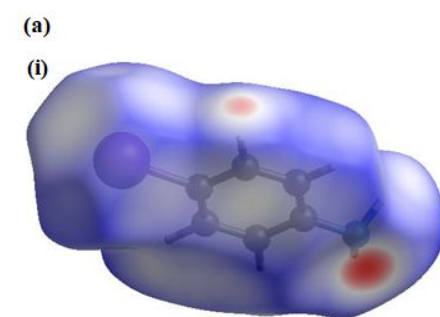

(b)

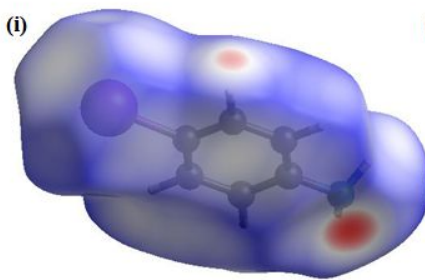

(c)

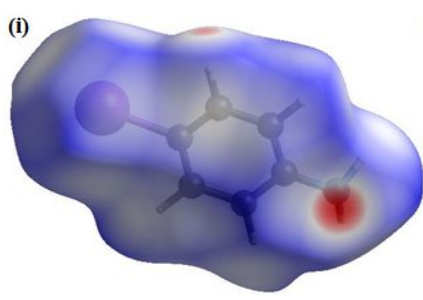

(ii)

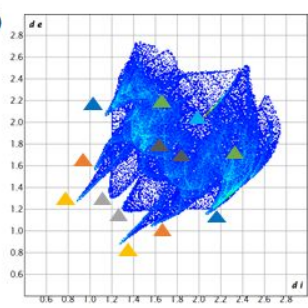

(ii)

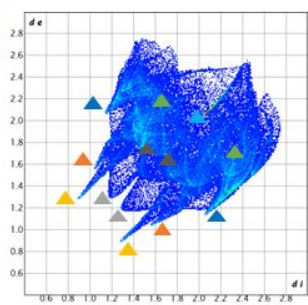

(ii)

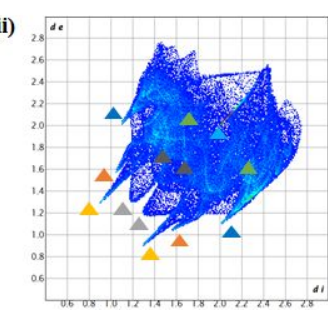

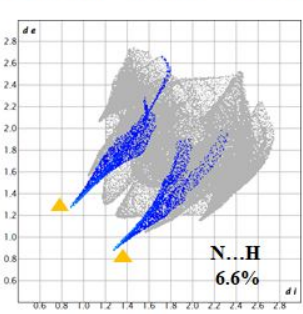
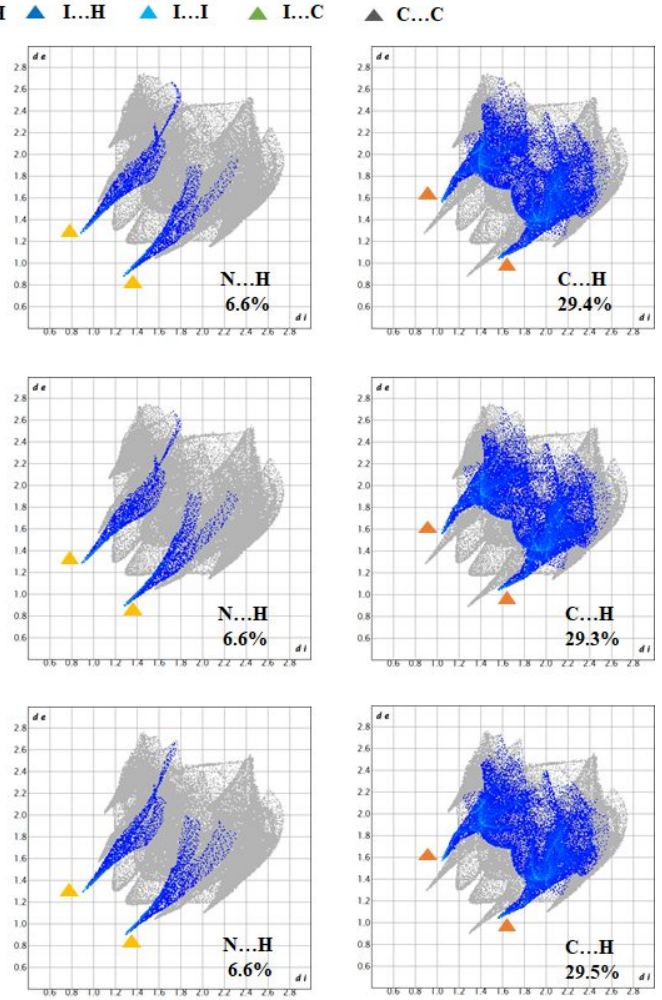

Figure S5. (a) Relative contributions of intermolecular interactions to the Hirshfeld surface area and its respective fingerprint plots of 4-Iodoaniline. (a) Mother domain $\left(\alpha_{M}\right)$, (b) Daughter domain $\left(\alpha_{\mathrm{D} 1}\right)$, and $(\mathbf{d})$ Daughter domain $\left(\alpha_{\mathrm{D} 2}\right)$. 
(a)

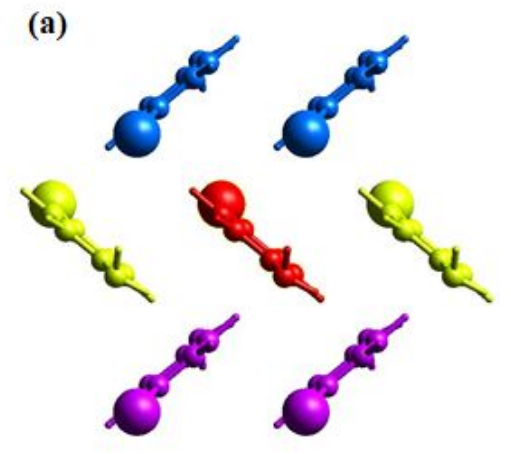

(b)
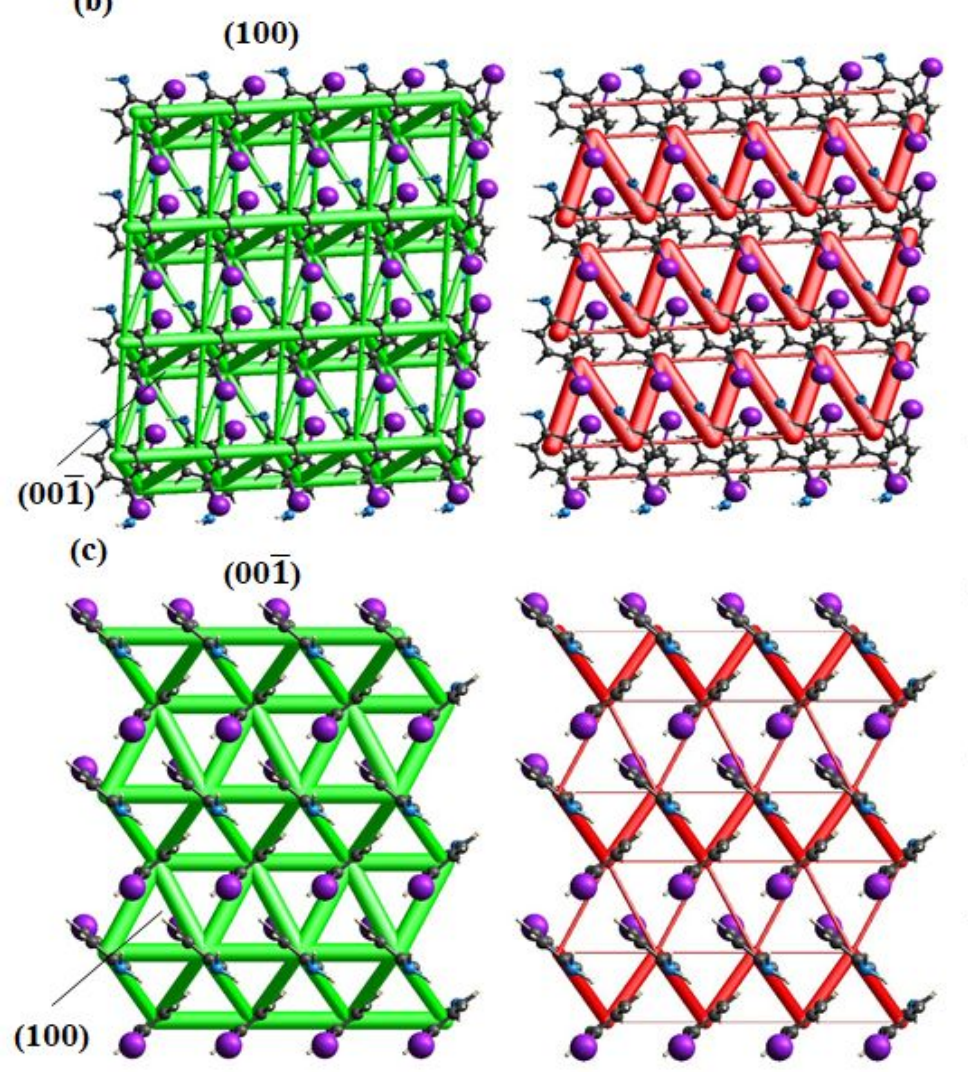
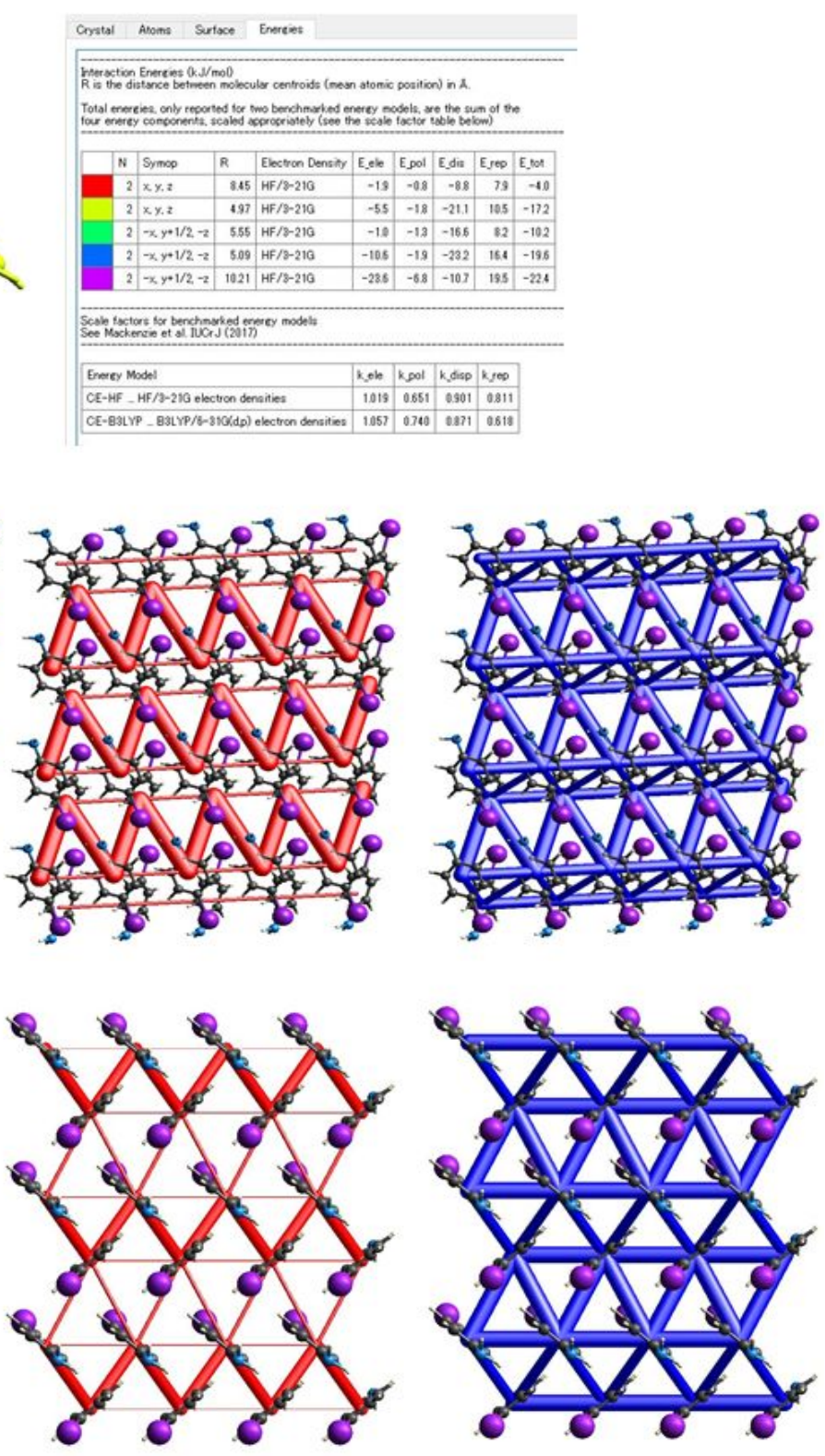

Figure S6. (a) Molecular structure pairs and the interaction energies $(\mathrm{kJ} / \mathrm{mole})$ obtained from energy framework calculation (b) Energy frameworks along $(0 \overline{2} 1)$ direction $\alpha_{M}$ to $\alpha_{D 1}$ and (c)

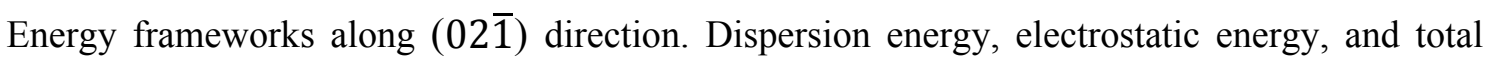
interaction energy are colored in green, red, and blue, respectively. (The energy scale factor, 25; the energy threshold, $5 \mathrm{~kJ} \mathrm{~mol}^{-1}$.) 